\title{
Detailed Analysis of Unified Power Flow Controller- A Review
}

\author{
Umar Hanfi \\ M.Tech Scholar \\ Truba Institute of Engineering \& Information \\ Technology \\ Bhopal, M.P., India \\ umarhanfi@gmail.com
}

\author{
Prof. Shravan Vishwakarma \\ Assistant Professor \\ Truba Institute of Engineering \& Information \\ Technology \\ Bhopal, M.P., India
}

\begin{abstract}
Renewable techniques have become more appealing for producing energy in village and moderately village regions because they reduce degradation of environment and provide a plentiful supply of free and environmental energy. Several research works have been carried out all over the planet to assess and investigate the feasibility of hybrid renewable resources (combination of two or more) with the electricity generation for usage in diverse implementation and livelihoods. An explanation of photovoltaic renewable energy systems is demonstrated in this paper.
\end{abstract}

Keywords: Renewable Energy, Hybrid system. UPFC, Solar PV system

\section{INTRODUCTION}

To provide energy to various load centers, modernized electrical systems are invented to work at a maximum reliability. Solar energy is a long-term, renewable energy source that will last for millions and millions of years until the sun sets for ever. The grid is linked up to massive solar parks. The most significant technological advances in recent years are FACTS (Flexible Alternating Current Transmission System) controls, as they enhance power transmitting and controlling ability of the system and its capacity [1]. With ever raising demands for electricity has ushered in novel developments in power production, such as the widespread utilization of renewable resources. The massive raise in load demands has necessitated the development of novel approaches for obtaining entry to distributed generators (DG).Besides that, engineers were the first to utilise ecofriendly energy supplies, owing to environmental impacts. Photovoltaic systems, wind based turbines, energy from tides, hydro-electricity, and other renewable energies are among them. The use of photovoltaic (PV) solar energy is speedily growing globally. Everywhere around globe, photovoltaic ( $\mathrm{pv}$ ) systems are growing at a rate of about $40 \%$ per year. Solar power-plants are intertwined with traditional power plants. Many developments of capacities of 9,500 MW, $500 \mathrm{MW}$, and other sizes have indeed been built around the globe. The large - scale implementation of solar power generators during traditional low generation times can be problematic. It is due to traditional generation's power amplification may be inadequate because of minimizes inertia, and power circulation trends may fluctuate greatly, imperiling the system's synchronization capability [2].
With regards to the system stability and its reliability, the traditional alternating current network has important benefits. Even so, due to limited controlling ability, the system is not being able to effectively control overcrowding on large transmission lines. Some lines, for e.g., are heavily loaded, whereas others are barely loaded. Undesirable loop circulations are also commonly encountered. This suggests not only ineffectual energy market working perfectly, but also the initial line to attain its capacity limitations, the entire system's capacity, even though the majority of the system's lines are less than their capacity limitations [3]. Because of the dual problems of global warming and depletion of natural resources, the upsurge of wind energy and solar energy has been recognized as a global awareness.With regards to voltage controlling, frequency controlling, transient stability, as well as vibrational damping, moreover, intermittent power through wind and solar resources has an adverse influence on the mesh network.

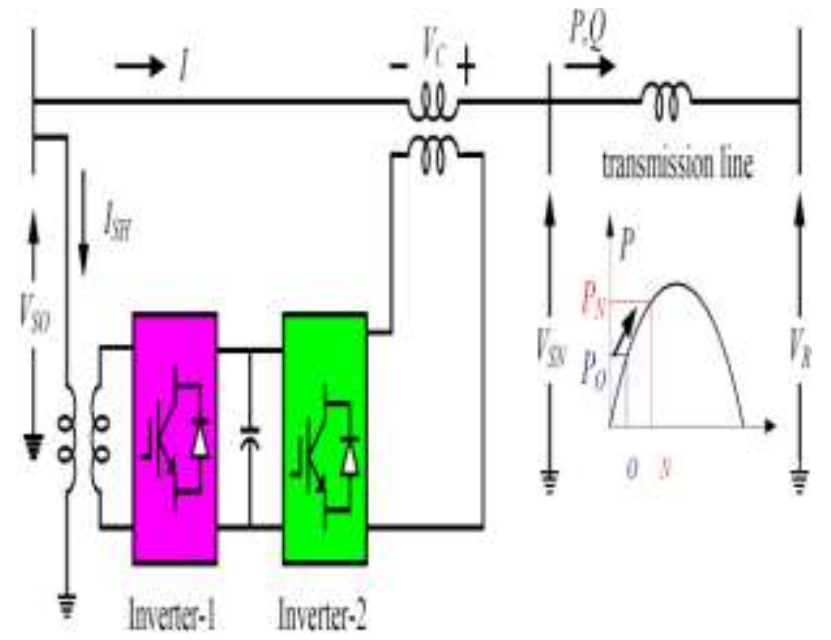

Figure 1 The Conventional UPFC

Thus there is a severe technological problem across the AC mesh grid's weaker controlling ability and the grid's decentralized, unorganized, and aperiodic wind energy and solar energy. Series B inductance or capacitor, Phase Shifting Transformer, Static Synchronous Compensator (STATCOM), Serial Static Synchronous Compensator (SSSC), \& Uniform Power Flow Controller (UPFC) are some of the power circulation control techniques existed for real world implementations. As seen in Fig. 1, an ideal FACTS 
device is a perfect blend of a STATCOM and an SSSC that shares common DC link. It allows you to control the circulation of real and reactive power on a line with thorough flexibility and control.This is the most efficient and useful FACTS system, deserving of 1) voltage controlling, 2) line impedance compensation, 3) phase-shifting, 4) dynamic damping of power fluctuations, and 5) voltage, impedance, phase, and damping control all at the same time. Conventional UPFC has been utilized in an array of applicants where an interface transformer is required to differentiate each inverter out from the transmission line.

\section{UNIFIED POWER FLOW CONDITIONER (UPFC)}

The UPFC is made up of two back-to-back converter topologies, Voltage Source Converter1 and Voltage Source Converter2, that are powered by a DC link offered by a dc storing capacitor. Such configurations function as an ideal ac to ac converter, wherein real power can flexibly circulate within each direction among the two converters' ac nodes, and every converter can start generating or absorbing reactive power autonomously as its own ac output node.

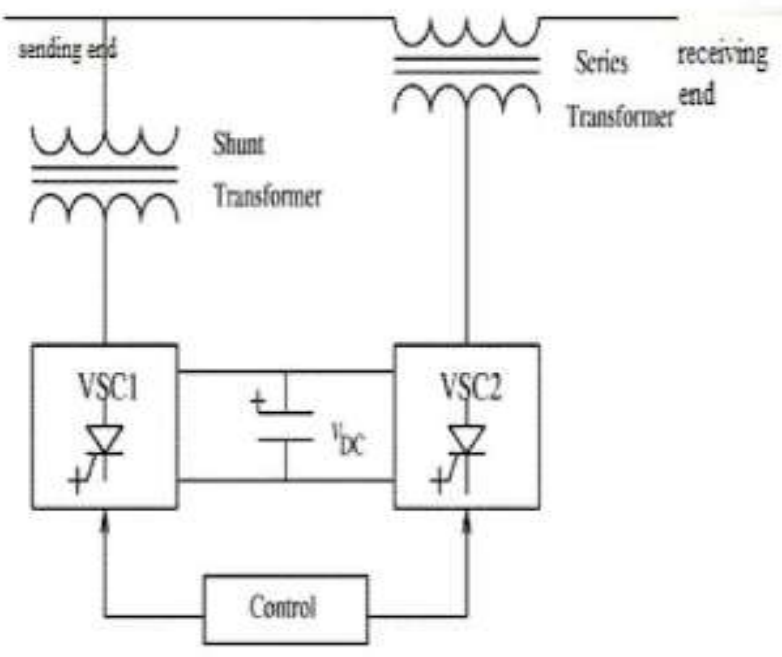

Figure 2 Basic Arrangement of UPFC

One Voltage Source Converter is wired in series to the transmission line via a series transformer, while another is linked in shunt to the transmission line via a shunt transformer. Two Voltage Source Converters DC terminals are combined, forming a route for active power interaction among the converters. Voltage Source Converter performs the core purpose of UPFC through injecting a voltage in series with the line with a manageable magnitude and phase angular position via an infusion transformer. This voltage injection serves as a sequential ac voltage source. The voltage source receives the transmission line current, actually results in an real power and reactive power interchange among it as well as the ac system. The converter generates the reactive power swapped at the dc terminal from within. The ac terminal's active power is converted to dc power, that also emerges as an actual power demand on the dc link. And Voltage Source Converter1 is responsible for supplying or absorbing the real power required by Voltage Source Converter 2 at the commonly shared dc link in supporting the real power sharing caused by the series voltage infusion. Voltage Source Converter1 converts Voltage Source Converter2's dc link power demand to ac and couples it to the transmission line bus via transformer which is linked in shunt. Furthermore, if needed, Voltage Source Converter1 can start generating or absorb easily controlled reactive power, providing freely shunt reactive compensation for the line.Therefore, Voltage Source Converter1 can be managed to function at a unity power factor or to transfer reactive power with the line unbiased of the reactive power transferred by VSC1. There can't be any reactive power flowing through the UPFC dc link, undoubtedly. The Merged FACTS category of devices involves the Unified Power Flow Controller (UPFC). A basic consistent relation is used to link up two three-phase converters in sequence. The UPFC's primary objectives is taken over by the serial converter. The serial transducer presents the desired voltage, amplitude, as well as angle into the line, thereby controlling the circulation of energy through everything. The shunt converter facilitates the serial converter through supplying the necessary actual power via the transitional circuit capacitor. Simultaneously, the shunt converter offers easily controlled current at the connector, allowing the voltage profile to be controlled close to 1 p.u . A stationary series synchronous compensator (SSSC) is being utilized in the serial converter, and a static synchronous compensator is utilized in the shunt converter (STATCOM).

A self-sufficient power grid depending on renewable energy can be a highly recommended and cost-effective remedy for remote areas and isolated societies, as grid advancement is also unworkable owing to socioeconomic and technical constraints. Because of their dependability, cost-effective setup, incredibly simple begins, compressed power-density, and flexibility, diesel generators were mostly frequent and utilized as a stand - alone power system implementation in remote and isolated regions. Increasing p rice fluctuations, on the other hand, make procedure prohibitively expensive. They also destroy the environment significantly.Renewables sources including wind and solar can offer clean and cheap energy over more remote regions. Because renewable energies are infrequent, hybrid pairings of multiple energy sources and energy storage can enhance the reliability as well as ensure ongoing, cost-effective power. Various types of energy manufacturing can be combined to increase energy effectiveness and enhance performance of a systems.

\section{LITERATURE REVIEW}

(Haque, Ali, \& Wolfs, 2020) [11] For Low Voltage supply voltage control as well as current balancing implementations in power-plants, this author recommends a UPFC $4 \mathrm{~L}$ configuration with shortened DC link throughput. The $4 \mathrm{~L}$ UPFC system's structure and composition, expense, and serviceability are improved dramatically by using a simple DC link capacitor rather than a massive electrolytic capacitor. The simulation results indicate that the developed voltage control methodology enables the 4L-UPFC gadget to control the output voltage at the control's end by providing offsetting 
voltages. The 4L-Unified Power Flow Control compensates for zero current on the power injectors at input stage.The compressed 4L-UPFC is being utilized as a post-top setup in Low Voltage distribution power supplies to overcome issues related to grid quality, that can save money. This machine inhibits OLTCs from being turned on and off frequently, thereby prolonging the life of OLTCs and allocation transformers.

(Xu et al., 2020) [12] This article presents a novel UPFC model approach depending on stochastic two-step coding that accounts for the maximum penetration level of renewables in transmission grids. The case illustrates that the proposed estimation process generates only small mistakes with little influence on ideal UPFC development planning however a substantial enhancement in computational effort. Benders' depending on decomposition solution methodology can also manage UPFC planning for massive power systems that are implicated in a range of functional situations. This difficult computational issue, but even so, cannot be dealt using traditional heuristic algorithms. Unified Power Flow Controller devices can be implanted in transmitting system to minimize power losses, voltage variations, and enhance system load capacity as a hopeful technological solution. The developed stochastic planning scheme has lower overheads than the predetermined masterplan, which include power dissipation expenses and UPFC planning expenses, even if UPFC planning expenses are increasing.

(Khurana\&Titare, 2020) [13] In this manuscript, an innovative control system, including a neural network, that is used to analyse UPFC. Artificial intelligence encompasses all of this. Artificial Neural Network is used in power circulation implementations to enhance overall system performance and minimize errors. The neural network is the most crucial consideration, and its specificity is inverse proportion to the prediction error. The Outcomes and Assessment section presently yields a more accurate reading than prior UPFC system conceptual design.

(Kalyan, 2021) [14] This article describes how to use a PIDD controller in conjunction with GWO to stabilise the frequency and voltage of a dual-zone system that is interrelated. In addition, the PIDD's superiority is validated by comparing its performance to that of the PI, PID, and PIDN control systems for a $0.01 \mathrm{MW}$ variability in Zone 1 load. Eventually, the GWO depending PIDD control system and UPFC-SMES control strategy are found to become the most efficient in keeping stable operation during large disturbances.

(Paital et al., 2021) [15] The efficiency of a UPFC relying on a comprehensive HHO-matched dual IT2FLL to reduce LFOs in the power supply system and improve system stability was investigated in the present study. The HHO method was used to optimise the control system parameters to enhance the stability, and the suggested double UPFC relying on IT2FLL was established. To overcome optimization difficulties and challenges, the HHO method enhances effectiveness and helps to balance its stages. Across time domain computer models and frequency domain illustrations including locus, Bode diagram, and Nyquist diagram, the effectiveness of the Cis-UPF relying on the dual IT2FLL was assessed on a SMIB system and a 3-zone-6 equipment energy system. In order to accomplish the structural analysis, real-time validation was conducted using OPAL-RT in additament to the simulation outcome.

(Haque, Ali, Wolfs, et al., 2020) [16] This article suggests enhanced sequence depending on control techniques for a UPFC 4L's series and shunt converters to control AC load voltages while preserving $2 \mathrm{HV}$ to eliminate the DC link out from 4L-UPFC. The AC load voltage is corrected with a controlled PS current, whereas the $2 \mathrm{HV}$ component is removed from the 4L-intermediate UPFC's circuit capacitor with a controlled LV current. The computation and test outcome demonstrate that the introduced sequence depending control techniques, when implemented to the $4 \mathrm{~L}-$ UPFC prototype's series and shunt converters, allow controllability of the load voltage whereas the removing $2 \mathrm{HV}$ out from UPFC's intervening circuit excluding additional compensation. Circuit. To construct an implementation of DC link capacitor dimensions in connection to the drive's able to operate voltages and currents, a relationship structure has been generated.

(Song et al., 2017) [17] This article presents a novel control system for the variety of Unified Power Flow Controller inverters in order to minimize line losses while also ensuring conditions in distribution networks including a high renewable energy content. The control structure is depending on the condition of minimal level line loss, as well as the security restrictions that have been derived and the techniques that have been suggested to address the diminished OPF issues. This study's control scheme demonstrates how the OPF resulting values can be merged with a dynamic control system to accomplish quick and optimal control. The removal of equality constraints as a way of reducing the computation complexity of OPF estimations is also mentioned.

(Park \& DeMarco, 2019) [18] In ability to properly explore their impacts inside the transmission, this article detailed sophisticated power grid modelling for accurate portrayal of power stations in elevated DER insertion scenarios based on the breaker node model and UPFC equipment. STF can help you build a network. Including decision variables affiliated to WPP as well as UPFC, the ACOPF framework developed using the STF aims to optimize transmission system working. The STF eradicates tedious configuration computation for substation realignment evaluation and wants to avoid the complicated modification and beginnings of low impedance bypass to depict bypass voltage sources perfect for UPFCs due to its versatility in portraying elements of the network.

(Suresh et al., 2018) [19]This work was commissioned to explain the complete behaviour of the Wind energy conversion system in both normal and anomalous scenarios, as well as in the occurrence of a $120 \mathrm{KV}$ bus fault. There are also waves formed for normal and fault situations. The software used to simulate all of the task is MATLAB / SIMULINK. Since this wind based turbine necessitates 
reactive power from the grid, voltage fluctuations occur in the case of system breakdown. And the breakdown or increase in tension were inside the IEGC's safety majors. If the voltage fluctuation exceeds safe levels, FACTS devices must be used to compensate. FRT can be gotten better in this article by combining UPFC with WECS and making comparisons to IEGC and USGC (US Grid Code). The findings indicate that if the PCC excluded UPFC fails, the voltage fluctuations, so if the voltage fluctuations occur, the wind based turbine must be disengaged out from grid to prevent impeller destruction. The fluctuations in power to the PCC violates the grid code's safe working margins.

(Wang et al., 2020) [20] This paper describes a VSG-enabled Unified Power Flow Controller (UPFC) that can compensate for voltage ups and downs at the common crossing point while also offering a stable voltage be reference for integrated Renewable Energy systems. This paper also develops a comprehensive controlling strategies and a small signal assessment. To demonstrate the UPFC-main VSG's upsides, it is then compared to an equivalent synchronous static compensator (STATCOM) with centralized storing energy, which is also controlled by VSG control.Simulating a UPFC-Voltage Source Generator as well as a STATCOMVoltage Source Generator, that facilitate the network connection of a 100 MVA Renewable Energy system, ensures the confirmation of the envisaged modeling and control technique.

\section{WorkingApproaches of UPFC}

The UPFC can operate in a variety of modes. The shunt inverter, in particular, works by injecting an easily controlled current forward into transmission line. With regard to the line voltage, the above current includes two elements: a real or direct element that is in phase or exact reverse phase with the line voltage, and a reactive or quadrature element that is in quadrature. The necessity to maintain the series inverter's real power determines the real element instantaneously. Rather than, the quadrature element can be arranged to any intended base value (inductive or capacitive) inside the inverter's capability, allowing it to absorb or produce reactive power out from line. There are two methods for controlling the shunt inverter: The reference or base input is an inductor based or capacitor based VAR demand in VAR control technique. The shunt inverter control converts the Var reference into a shunt current request, then calibrates the inverter's gating to reach the required current. A closed loop system indicating the dc bus voltage, $\mathrm{Vdc}$, is also necessary for this type of method of controlling. Fully automated Voltage Control Method: The reactive current of the shunt inverter is autonomously controlled to uphold a reference or base value for the transmission line voltage at the connection point. Voltage closed loop signals are collected from the transmission end bus continue to feed the shunt coupling transformer for this method of controlling. To impact the power circulation on the line, the series inverter helps to control the magnitude and angle of the voltage infused in series with the transmission line. There are many methods for determining the actual value of the infused voltage. The benchmark input data are straightforwardly the angle of magnitude \& phase of the series voltage inside direct voltage insertion method. The reference input is phase dislocation among the transmitting and reception end voltages in Phase Angle Shifter Emulation mechanism. Impedance of a line Emulation mode: An impedance value to introduced in series involving the line impedance is the benchmark input. The benchmark inputs are values of $\mathrm{P}$ and $\mathrm{Q}$ to sustain on the transmission line notwithstanding the modifications in Automatic Power Flow Control Method.

\section{A. Shunt Controlled UPFC}

An UPFC's shunt controller functions similarly to a STATCOM. The primary objective is to charge the intervening circuit capacitor voltage, wherein the serial converter can use to control power circulation. It also keeps the voltage levels at the point of connection constant. Figure 2 depicts the controlling mechanism.

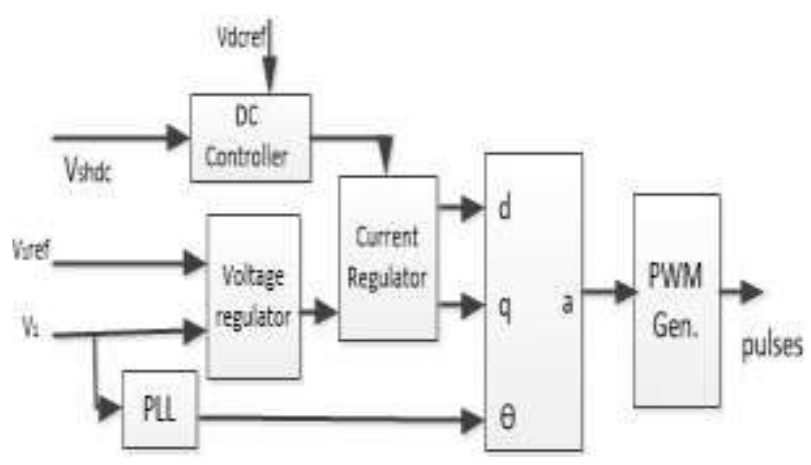

Figure 3 Block Diagram of Shunt Controlled UPFC

Vdcref and Vref (preferred operating points) are accepted as reference or base values. A conventional PI controller that supports Idref regulates the error that happens among Vdcref and the straightforward bypass voltage (Vshdc). A very well PI controller also generates the Iqref, which adapts the error between the target position and the main supply voltage element.

\section{B. Series Controller}

Similar vector current control scheme is used in the series controlling approach. Figure 3 depicts the control mechanism. The primary function of the series converter is to develop the accurate active power and reactive power on the line by using the intermediate circuit capacitor voltage. 


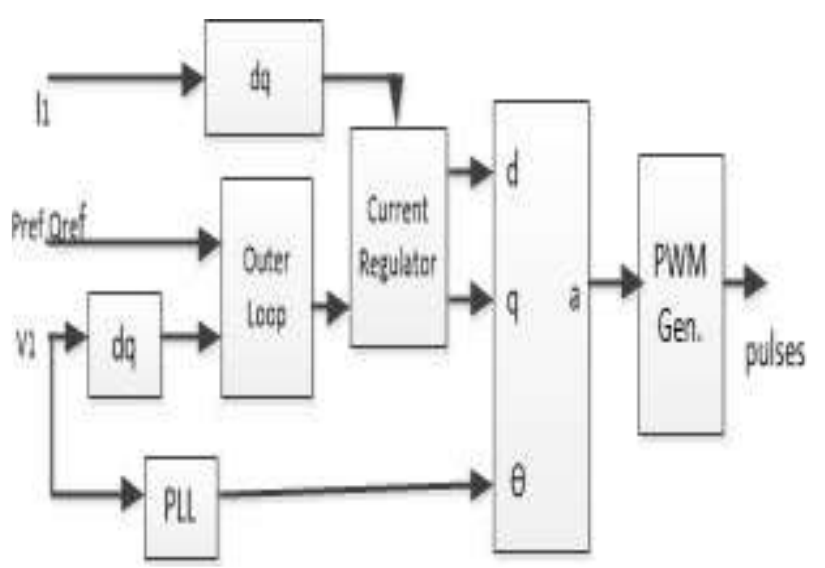

Figure 4 Block Diagram of Series Controlled UPFC

It primarily uses Pref and Qref as reference or base values. Employing the PI equaliser, compare Pref with $\mathrm{Vd}$, grid and Qref with $\mathrm{Vd}$, grid to acquire Idref and Iqref. The following are the derivations that carry out the aforementioned procedure:

$$
\begin{gathered}
I_{d r e f}=\frac{V_{d r e f} * P_{r e f}}{\sqrt{\left(V_{d}\right)^{2}+\left(V_{q}\right)^{2}}}+\frac{V_{q} * Q_{r e f}}{\sqrt{\left(V_{d}\right)^{2}+\left(V_{q}\right)^{2}}} \\
I_{d r e f}=\frac{V_{d r e f} * P_{r e f}}{\sqrt{\left(V_{d}\right)^{2}+\left(V_{q}\right)^{2}}}-\frac{V_{q} * Q_{r e f}}{\sqrt{\left(V_{d}\right)^{2}+\left(V_{q}\right)^{2}}}
\end{gathered}
$$

\section{Photo-Voltaic System}

Article [9] presents the modelling and regulation of the hybrid approach, that involves Photovoltaic system, wind energy, and battery system. Performance parameters of hybrid systems vary including solar radiation, temperature, and speed of wind, and performance parameters are inherently unstable. Monitoring the electricity transmission in a grid-connected system is crucial. The person in charge of various events seeks to boost the transmission line's capacity. Among Bthe various fact controllers, Unified Power Flow Converter generates total control over the power grid. teA photovoltaic system, a wind based turbine, a battery accumulator, a DC-DC converter, a voltage regulator, and a UPFC regulator are all included in this prototype. Researchers use MPPT technologies to boost the output voltage including both power sources and DC-DC inverter to accomplish a variety. The voltage regulator is built in to regulate the inverter's pulse, that also transform the produced direct current to alternating current. The dilemma of raising the output voltage of a hybrid system connected to the grid to enhance organizational power quality is minimized with UPFC.

\section{CONCLUSION}

The work here presents a solar-based renewable energy system. The solar plant was derived in connection with the construction of the grid with UPFC in the load line. We have discussed about the unified power flow controller, its principle of working. We have also highlighted the work done by researchers to enhance the performance of UPFC. Further we have also shown the shunt and series controllers which are inbuilt in the Unified power flow controller. The system was also hybridized with micro-hydro technology in future work. The standard design of the UPFC could not stabilize the system and thus we design a hybrid energy system with a compensator in our further research.

\section{References}

1) Ramya, L. (2016). as PV-UPFC for the Enhancement of Transient Stability in Power Grid.

2) Jain, K., Bhargava, A., \&Mewara, B. (2018). Power Quality Enhancement Using Unified Power Flow Controller in Standalone Grid Connected Solar PV System. Proceedings of the International Conference on Inventive Research in Computing Applications, ICIRCA 2018, Icirca, 709-713. https://doi.org/10.1109/ICIRCA.2018.8597287

3) Peng, F. Z., Liu, Y., Yang, S., Zhang, S., Gunasekaran, D., \&Karki, U. (2016). Transformer-less unified powerflow controller using the cascade multilevel inverter. IEEE Transactions on Power Electronics, 31(8), 54615472. https://doi.org/10.1109/TPEL.2015.2497078

4) Yadav, R., \&Sheoran, S. K. (2020). Autoregressive Model for Multivariate Crime Prediction. https://doi.org/10.1007/978-981-15-3643-4_23

5) Kopperundevi, P., \& Surya Prakash, M. (2021). An efficient hardware architecture for deblocking filter in HEVC. In Lecture Notes in Electrical Engineering (Vol. 661). https://doi.org/10.1007/978-981-15-4692-1_46

6) Sen, A., Banerjee, A., \&Nannam, H. (2019). A Comparative Analysis between UPFC and DPFC in a Grid Connected Photovoltaic System. IEEE International Conference on Intelligent Techniques in Control, Optimization and Signal Processing, INCOS 2019, 1-5. https://doi.org/10.1109/INCOS45849.2019.8951352

7) Nahak, N., Sengupta, P., \&Mallick, R. K. (2019). Improvement of small signal stability of solar integrated power system by UPFC based optimal controller. 2019 Innovations in Power and Advanced Computing Technologies, i-PACT 2019, 1-6. https://doi.org/10.1109/i-PACT44901.2019.8960077

8) Joglekar, J., \&Nerkar, Y. (2010). Application of UPFC for improving micro-grid voltage profile. 2010 IEEE International Conference on Sustainable Energy Technologies, ICSET 2010, 1-5. https://doi.org/10.1109/ICSET.2010.5684408

9) Chaudhary, V., Bhargava, A., \&Verma, S. (2019). Power quality enhancement using unified power flow controller in grid connected hybrid PV/Wind system. Proceedings of the 4th International Conference on Communication and Electronics Systems, ICCES 2019, Icces, 20642069.

https://doi.org/10.1109/ICCES45898.2019.9002306 
10) Zhang, S., Lu, X., Liu, Y., Ge, B., \& Peng, F. Z. (2013). Nine IGBTs based UPFC topology and control for renewable power integration. Conference Proceedings IEEE Applied Power Electronics Conference and Exposition - APEC, 1294-1300. https://doi.org/10.1109/APEC.2013.6520466

11) Haque, M. M., Ali, M. S., \& Wolfs, P. (2020). A UPFC Topology for LV Feeder Voltage Regulation and Current Balance in High PV Penetration Applications. 2020 IEEE International Conference on Power Electronics, Smart Grid and Renewable Energy, PESGRE 2020, 1-6. https://doi.org/10.1109/PESGRE45664.2020.9070741

12) Xu, X., Jia, Y., Xu, Z., \& Li, J. (2020). Optimal Unified Power Flow Controller Planning in Transmission Grids with Uncertainty Consideration. 2020 IEEE/IAS Industrial and Commercial Power System Asia, I and CPS Asia 2020, 1689-1695. https://doi.org/10.1109/ICPSAsia48933.2020.9208445

13) Khurana, B. V., \&Titare, L. S. (2020). Improvement of power flow and voltage stability using UPFC with artificial neural network in Matlab. Proceedings - 2020 IEEE International Symposium on Sustainable Energy, Signal Processing and Cyber Security, ISSSC 2020, 5-8. https://doi.org/10.1109/iSSSC50941.2020.9358842

14) Kalyan, C. N. S. (2021). UPFC and SMES based Coordinated Control Strategy for Simultaneous Frequency and Voltage Stability of an Interconnected Power System. ICPEE 2021 - 2021 1st International Conference on Power Electronics and Energy. https://doi.org/10.1109/ICPEE50452.2021.9358576

15) Paital, S. R., Ray, P. K., \&Mohanty, S. R. (2021). A robust dual interval type-2 fuzzy lead-lag based UPFC for stability enhancement using Harris Hawks Optimization. ISA Transactions, xxxx. https://doi.org/10.1016/j.isatra.2021.05.029

16) Haque, M. M., Ali, M. S., Wolfs, P., \&Blaabjerg, F. (2020). A UPFC for Voltage Regulation in LV Distribution Feeders with a DC-Link Ripple Voltage Suppression Technique. IEEE Transactions on Industry Applications, 56(6), 6857-6870. https://doi.org/10.1109/TIA.2020.3023068

17) Song, P., Xu, Z., Dong, H., Cai, H., \&Xie, Z. (2017). Security-constrained line loss minimization in distribution systems with high penetration of renewable energy using UPFC. Journal of Modern Power Systems and Clean Energy, 5(6), 876-886. https://doi.org/10.1007/s40565-017-0334-8

18) Park, B., \& DeMarco, C. L. (2019). Advanced modeling of DERs and UPFC devices with Sparse Tableau Formulation for ACOPF. Electric Power Systems Research, 174(March), 105870. https://doi.org/10.1016/j.epsr.2019.105870

19) Suresh, H. L., Sharanappa, M., \& Ramesh, C. H. V. (2018). Performance of Wind Energy Conversion
System during Symmetrical fault Power Quality Improvement of Grid-Connected WECS by UPFC. 2018 4th International Conference for Convergence in Technology, I2CT 2018, 2-7. https://doi.org/10.1109/I2CT42659.2018.9058230

20) Wang, S., Jing, L., Zhao, Y., Wickramasinghe, H. R., Wu, X., \&Konstantinou, G. (2020). Operation of unified power flow controller as virtual synchronous generator. IEEE Access, 8, 162569-162580. https://doi.org/10.1109/ACCESS.2020.3021388

21) Sen, A., Banerjee, A., \&Nannam, H. (2020). A comparative analysis between two DPFC models in a grid connected Hybrid Solar- Wind Generation system. 2020 IEEE International Conference on Power Electronics, Smart Grid and Renewable Energy, PESGRE 2020, 1-6. https://doi.org/10.1109/PESGRE45664.2020.9070373

22) Saberi, H., Mehraeen, S., \& Wang, B. (2018). Stability improvement of microgrids using a novel reduced UPFC structure via nonlinear optimal control. Conference Proceedings - IEEE Applied Power Electronics Conference and Exposition - APEC, 2018-March, 32943300. https://doi.org/10.1109/APEC.2018.8341575 